\title{
INELASTIC COLLISIONS OF INTERSTELLAR MOLECULES
}

\author{
Experiment versus Theory
}

\author{
J.J. TER MEULEN \\ Department of Molecular and Laser Physics \\ University of Nijmegen \\ Toernooiveld 1, 6525 ED Nijmegen, The Netherlands
}

\begin{abstract}
The rotational energy transfer of $\mathrm{NH}_{3}, \mathrm{OH}$ and $\mathrm{D}_{2} \mathrm{CO}$ in inelastic collisions with $\mathrm{He}$ and $\mathrm{H}_{2}$ is studied in a crossed molecular beam experiment. The molecules are prepared in a single initial state by rotational cooling in an adiabatic expansion followed by electrostatic state selection. Relative state-to-state cross sections are determined by measuring the collision induced redistribution of the population of the initial state by using state selective laser detection techniques. The results for $\mathrm{NH}_{3}$ and $\mathrm{OH}$ are compared to theoretical values obtained from quantum calculations. Except for $\mathrm{NH}_{3}-\mathrm{He}$ where theory predicts a parity selection rule for transitions to the $3_{3}$ and $4_{3}$ states, which is not observed in the experiment, good agreement between experiment and theory is obtained.
\end{abstract}

\section{Introduction}

Inelastic collisions with $\mathrm{H}_{2}$ or $\mathrm{He}$ form a dominant process in the rotational energy transfer of interstellar molecules. Knowledge of the state-to-state collision cross sections is needed to explain the observed anomalous nonthermal or maser radiation. In most models either theoretical values for these cross sections are used or experimental data on averaged collision rates since state-to-state cross sections are usually not available. The rates obtained from line broadening or pump-probe experiments involve, however, a manifold of inelastic transitions from or to the probed energy state. The determination of a specific state-to-state cross section requires conditions which are not easily met in a conventional gas cell experiment. First the initial state must be earmarked, secondly not more than one single collision should take place, and thirdly the collision induced redistribution of population has to be probed by a state selective detection technique. The 


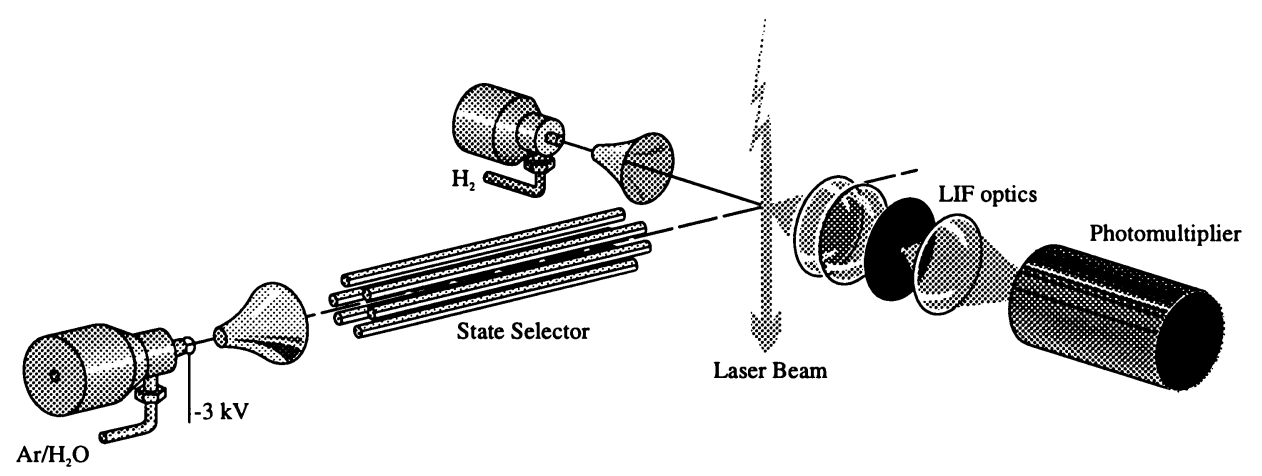

Figure 1. Artists impression of the crossed molecular beam experiment. The setup shown is for $\mathrm{OH}$, but is basically the same for $\mathrm{NH}_{3}$ and $\mathrm{D}_{2} \mathrm{CO}$.

last requirement is easiest to fulfill by the use of sensitive laser detection techniques, such as LIF (laser-induced-fluorescence) or REMPI (resonanceenhanced multi-photon ionization) detection. With respect to the other two conditions, the preparation of a single initial state and the occurrence of only a single collision event, these can in principle be met in a crossed molecular beam experiment. The density of the $\mathrm{He}$ or $\mathrm{H}_{2}$ (secondary) beam is then adjusted such that the molecules of the other (primary) beam typically undergo on the average 0.2 to 0.3 collisions. The preparation of the initial state is achieved by rotational cooling in the adiabatic expansion of the primary beam. A first crossed molecular beam experiment on $\mathrm{OH}$ radicals was reported by Andresen et al. (1991) in which the rotational energy transfer from the $J=3 / 2$ ground state was studied. Since both $\Lambda$ doublet states were prepared the results are relative cross sections averaged over the two doublet states. Recently we reported a crossed beam experiment on $\mathrm{OH}-\mathrm{Ar}$ collisions in which the molecules in the lower $J=3 / 2$ doublet state (of $e$-symmetry) are deflected out of the beam by an electrostatic state selector (Schreel et al., 1993; Schreel et al., 1996). As a result $\Lambda$-doublet state resolved cross sections are obtained for transitions from the upper $\Lambda$-doublet state (of $f$-symmetry). Similar experiments were performed before on $\mathrm{NH}_{3}$ (Schleipen \& Ter Meulen, 1991; Schleipen et al., 1992; Schleipen et al., 1993) and $\mathrm{H}_{2} \mathrm{CO}$ (Van Hulst et al., 1987a; Van Hulst et al., 1987b). In these experiments the electrostatic state selection was applied for para- $\mathrm{NH}_{3}$, where the ground rotational state is the $J_{K}=1_{1}$ inversion doublet, as well as for ortho- $\mathrm{H}_{2} \mathrm{CO}$, with the $J_{K}=1_{1} K$-doublet as the lowest state.

In this overview the results for the inelastic scattering of $\mathrm{OH}, \mathrm{NH}_{3}$ and $\mathrm{D}_{2} \mathrm{CO}$ by $\mathrm{He}$ and $\mathrm{H}_{2}$ are summarized. The formaldehyde isotope $\mathrm{D}_{2} \mathrm{CO}$ 
was chosen instead of $\mathrm{H}_{2} \mathrm{CO}$ because of a higher LIF detection efficiency by an order of magnitude. For $\mathrm{OH}$ and $\mathrm{NH}_{3}$ the measured cross sections are compared to theoretical values obtained from quantum calculations, which are mostly based on ab-initio intermolecular potentials. In case of $\mathrm{OH}$ also collisions with para- $\mathrm{H}_{2}$ will be considered.

\section{Experiment}

A schematic overview of the experimental set up is shown in Fig. 1. The crossed molecular beam machine is described in full detail elsewhere (Schreel et al., 1993; Schleipen \& Ter Meulen, 1991; Van Hulst et al., 1987b). Two molecular beams are crossed perpendicularly in a high vacuum chamber, using pulsed valves, except for the measurements on formaldehyde where both beams are continuous. The primary beam contains the $\mathrm{NH}_{3}, \mathrm{OH}$, or $\mathrm{D}_{2} \mathrm{CO}$ molecules seeded in Ar. The $\mathrm{OH}$ molecules are produced in an electrical discharge in an $\mathrm{H}_{2} \mathrm{O} / \mathrm{Ar}$ mixture at the exit hole of the pulsed valve. In the adiabatic expansion the molecules are cooled down to the rotational ground state, which except for ortho- $\mathrm{NH}_{3}$ is a doublet. In order to prepare only a single initial state the molecules in the lower doublet state are deflected from the beam axis. As a result a nearly pure single state distribution is obtained; in all measurements the fraction of molecules in the prepared initial state was between 90 and $95 \%$.

The rotational state distribution of $\mathrm{OH}$ and $\mathrm{D}_{2} \mathrm{CO}$ is probed by LIF at $308 \mathrm{~nm}$ and $325 \mathrm{~nm}$, respectively, whereas $\mathrm{NH}_{3}$ is detected by (2+1) REMPI via the $\tilde{B}, v^{\prime}=0$ and 1 states at $337 \mathrm{~nm}$ and $332 \mathrm{~nm}$, respectively. In case of $\mathrm{OH}$ and $\mathrm{NH}_{3}$ a pulsed laser beam is focused in the collision center and the produced photons (LIF) or ions (REMPI) are detected by a photomultiplier or a particle multiplier tube, respectively. The $\mathrm{D}_{2} \mathrm{CO}$ molecules are detected by a cw laser beam at $325 \mathrm{~nm}$ at a distance of $83 \mathrm{~mm}$ from the collision center. By rotating the probe area around the collision center differential cross sections are measured.

In all cases the observed signal is linearly proportional to the population of the probed state, which enables the direct determination of relative stateto-state cross sections. Elaborate checks upon the single collision condition have been performed. It can be shown that in case of multiple collisions the scattering signal is no longer linearly proportional to the secondary beam density (Schleipen \& Ter Meulen, 1991). All measurements were therefore performed at $\mathrm{He}$ and $\mathrm{H}_{2}$ densities far below the onset of a non-linear behaviour of the scattering signal. 

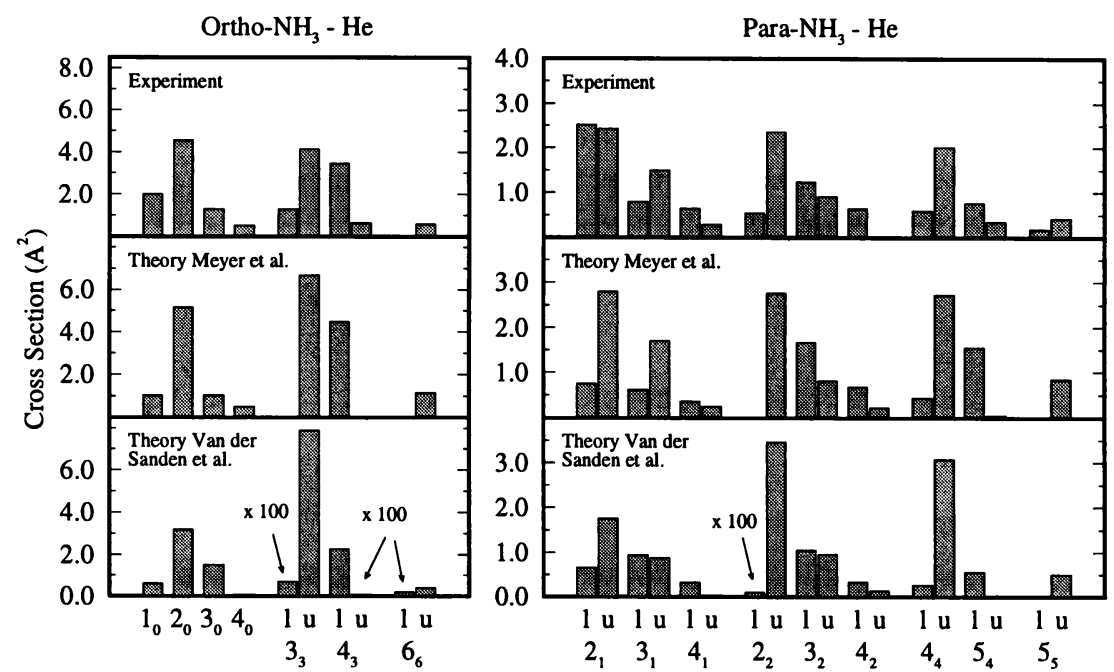

Figure 2. State-to-state cross sections for rotational energy transfer of ortho- $\mathrm{NH}_{3}\left(\mathrm{O}_{0}\right)$ and para- $\mathrm{NH}_{3}\left(1_{1} u\right)$ in collisions with $\mathrm{He}$. The experimental results are compared to theoretical values from Meyer et al. (1986) and van der Sanden et al. (1995).

\section{Experimental results and comparison with theory}

\section{1. $\mathrm{NH}_{3}$}

The cross sections for He- or $\mathrm{H}_{2}$-scattering of $\mathrm{NH}_{3}$ were measured for transitions with $|\Delta K|=0,3$ and 6 and with $\Delta J$ up to 6 . The collision energy is $435 \mathrm{~cm}^{-1}$ for $\mathrm{NH}_{3}-\mathrm{He}$ and $596 \mathrm{~cm}^{-1}$ for $\mathrm{NH}_{3}-\mathrm{H}_{2}$. From the measurements relative cross sections are obtained with an accuracy of $10 \%$. Absolute values are derived by scaling the experimental results to theoretical values in such a way that the sum of the cross sections for ortho- $\mathrm{NH}_{3}$, as well as for para- $\mathrm{NH}_{3}$, is the same for experiment and theory. The absolute data are listed in (Schleipen \& Ter Meulen, 1991).

\subsection{1. $\mathrm{NH}_{3}-\mathrm{He}$}

The experimental results for collisions with $\mathrm{He}$ are presented graphically in Fig. 2. The bars in Fig. 2 give the magnitude of the measured cross sections. For each excited $J_{K}$ state two bars are plotted for the two parity states, except for $K=0$. Also plotted in Fig. 2 are theoretical values calculated by Meyer et al. (1986) and Van der Sanden et al. (1995). Meyer et al. performed a Coupled States (CS) calculation at a collision energy of $789 \mathrm{~cm}^{-1}$ using an experimentally modelled intermolecular potential. Van der Sanden et al. did calculations at the experimental collision energy applying the more accurate quantum mechanical Close Coupling (CC) method 
using an ab-initio potential. As can be seen from the figure, apart from parity propensities, there is a reasonably good qualitative agreement between both calculations and the experimental results. For ortho- $\mathrm{NH}_{3}$ the cross section $\sigma\left(0_{0} \rightarrow 2_{0}\right)$ is considerably larger than $\sigma\left(0_{0} \rightarrow 1_{0}\right)$, as theory predicts. The experimental ratio of these cross sections of 2.3 is, however, noticeably smaller than the calculated ratio of 5.2. The transitions to the $3_{3}$ and $4_{3}$ states are relatively strong; when summing the cross sections for the transitions to the two parity states the experimental results are very close to the values calculated by Meyer et al. The calculations by Van der Sanden et al. seem to overestimate the sum of the cross sections for the transitions to the $3_{3}$ doublet and to underestimate the transitions to the $4_{3}$ doublet. Also with respect to the cross sections for para- $\mathrm{NH}_{3}$ the experimental parity summed values show a better agreement with the calculated values of Meyer et al. than with the results of Van der Sanden et al.

Strong deviations show up when considering the excitation of the two parity states belonging to a specific $J_{K}$ state. Whereas theory predicts strong parity propensity rules, particularly for transitions to the $3_{3}$ and $4_{3}$ states for ortho- $\mathrm{NH}_{3}$ and to the $2_{2}$ state for para- $\mathrm{NH}_{3}$, this is not observed in the experiment. For a similar situation observed for $\mathrm{NH}_{3}-\mathrm{Ar}$ (Schleipen et al., 1992) it was shown by Van der Sanden et al. (1992) that the complete absence of cross sections for transitions to the lower $3_{3}$ and the upper $4_{3}$ doublet state is an artifact of the CS approximation due to the neglect of Coriolis terms in the Hamiltonian. When applying the CC approximation, where these terms are taken into account, significant transition probabilities were obtained. However, when applying the $\mathrm{CC}$ approximation to the $\mathrm{NH}_{3}-\mathrm{He}$ system they found that these Coriolis terms have little influence resulting in still very small but non-zero cross sections, as can be seen in Fig. 2. The same authors also showed that the fact that the initial state preparation in the experiment is not perfect may cause non-negligible effects. States which are weakly populated, i.e. the $1_{0}$ state for ortho- $\mathrm{NH}_{3}$ and the lower $1_{1}$ state for para- $\mathrm{NH}_{3}$, may provide a significant contribution to otherwise small cross sections. Taking this into account the authors found a population transfer up to even $40 \%$ of the observed values for $\sigma\left(0_{0} \rightarrow 3_{3} l\right)$ and $\sigma\left(0_{0} \rightarrow 4_{3} u\right)$, which, however, still leaves a non-understood discrepancy between theory and experiment.

\subsection{2. $\mathrm{NH}_{3}-\mathrm{H}_{2}$}

In Fig. 3 the measured cross sections for transitions induced by collisions with $\mathrm{H}_{2}$ are presented. In the same figure also theoretical values of Ebel et al. (1990) and of Offer \& Flower (1990) are shown. The experimental values are scaled to the calculated values of Ebel et al. Strong differences with the $\mathrm{NH}_{3}-\mathrm{He}$ system can be noticed. For ortho- $\mathrm{NH}_{3}$ the transition 

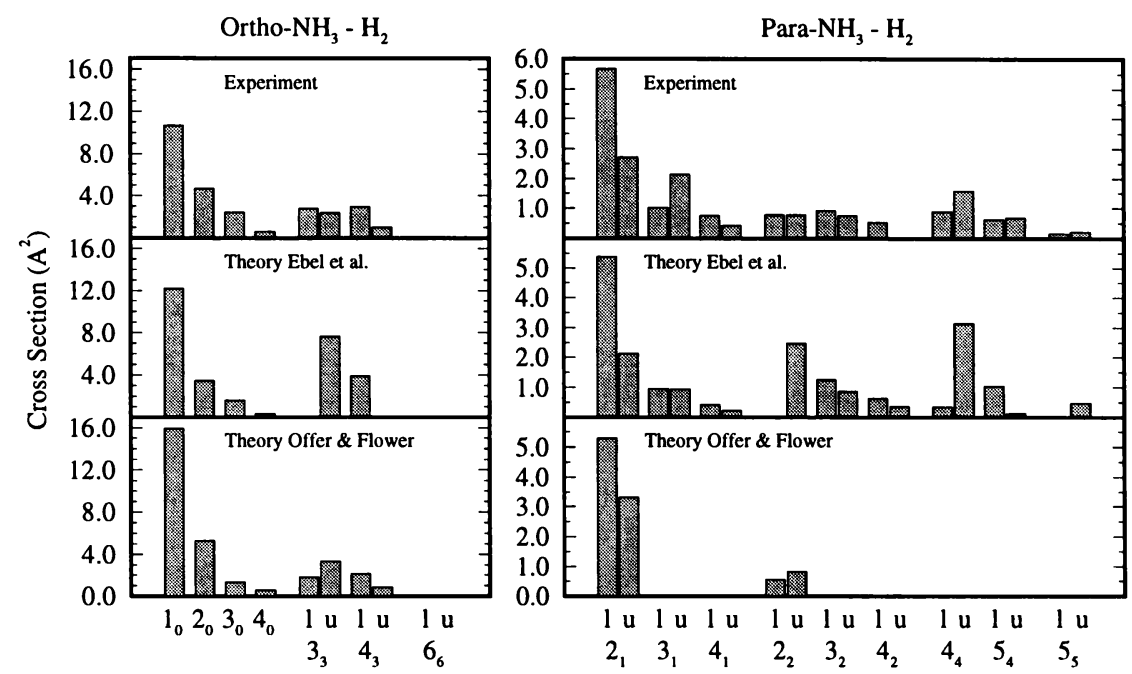

Figure 3. State-to-state cross sections for rotational energy transfer of ortho- $\mathrm{NH}_{3}\left(0_{0}\right)$ and para- $\mathrm{NH}_{3}\left(1_{1} u\right)$ in collisions with $\mathrm{H}_{2}$. The experimental results are compared to theoretical values from Ebel et al. (1990) and Offer \& Flower (1990).

to the $1_{0}$ state is now the strongest one, as predicted also by theory. For para- $\mathrm{NH}_{3}$ there is a strong preference for the excitation to the lower $2_{1}$ state, in agreement with theory. The parity propensities as observed in $\mathrm{He}$ collisions do not show up so strongly. When summing the cross sections for the transitions to the two parity states belonging to the same $J_{K}$ state a reasonably good agreement between experiment and theory is obtained. The calculations by Ebel et al. were performed in the CS approximation at a collision energy of $604 \mathrm{~cm}^{-1}$. As for He the cross sections for the transitions to the lower $3_{3}$ and upper $4_{3}$ states are per definition zero in this CS framework, whereas the transition to the lower $2_{2}$ state is extremely weak. Offer \& Flower performed CC calculations and expanded the potential in a more accurate way than Ebel et al. As a result they obtained relatively large cross sections for the transitions to the $3_{3} l, 4_{3} u$ and $2_{2} l$ states. Some deviations between experiment and theory are still present, which might be due to the presence of $\mathrm{H}_{2}(J=2)$ in the secondary beam, which is not taken into account in the comparison with theory (see the $\mathrm{OH}-\mathrm{H}_{2}$ results below).

\section{2. $\mathrm{OH}$}

The measurements on $\mathrm{OH}-\mathrm{He}$ and $\mathrm{OH}-\mathrm{H}_{2}$ collisions have been performed both in the presence and in the absence of the electrostatic state selector. In the first situation the initial state is the upper $(f) \Pi_{3 / 2}, J=3 / 2, \Lambda$ - 

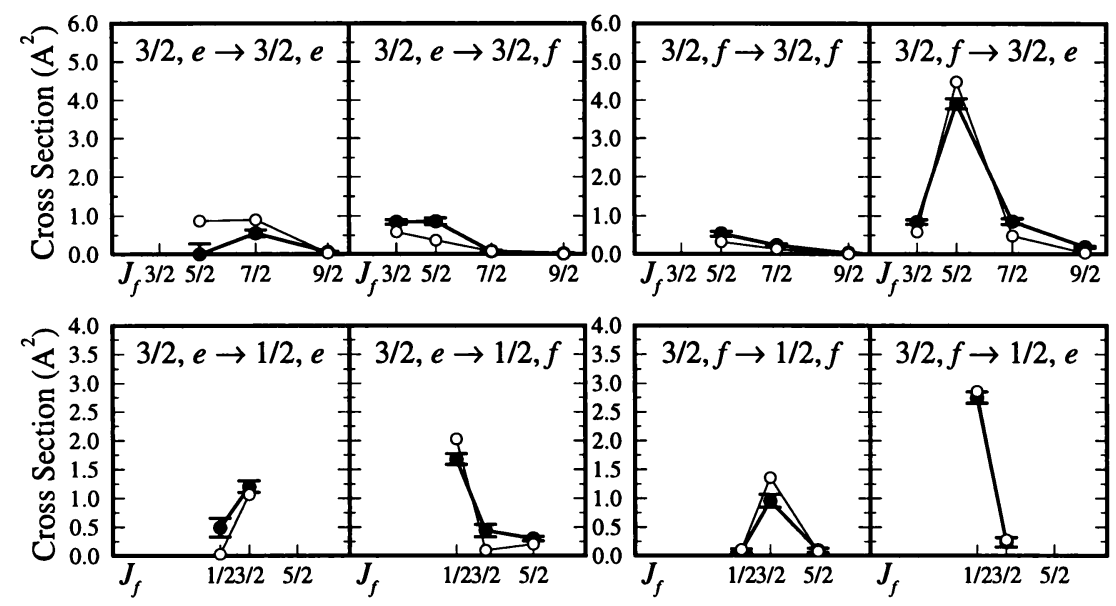

Figure 4. State-to-state cross sections for rotational energy transfer of $\mathrm{OH}$ $\left(\Pi_{3 / 2}, J=3 / 2\right)$ in the upper $(f)$ and lower $(e) \Lambda$-doublet state in collisions with He. The experimental results $(\bullet)$ are compared with theoretical $(\circ)$ values from Esposti et al. (1995).

doublet state, in the latter case both the upper and the lower (e) state are populated. By "subtracting" the results for the upper state the cross sections for transitions from the lower $\Lambda$-doublet state are obtained (Schreel et al., 1996). In addition to transitions within the $\Pi_{3 / 2}$ rotational ladder up to $J=9 / 2$ also spin-orbit changing transitions to the $\Pi_{1 / 2}$ ladder up to $J=5 / 2$ are studied. The collision energy is $394 \mathrm{~cm}^{-1}$ for He scattering and $595 \mathrm{~cm}^{-1}$ for $\mathrm{H}_{2}$ scattering. Details of the measurements and numerical values for the obtained cross sections are given in (Schreel et al., 1993) for $\mathrm{OH}-\mathrm{He}$ and in (Schreel et al., 1996) for $\mathrm{OH}-\mathrm{H}_{2}$.

\subsection{1. $\mathrm{OH}-\mathrm{He}$}

The measured cross sections are presented in Fig. 4. In the left hand side the results for transitions from the lower $J=3 / 2, \Lambda$-doublet state are shown, in the right hand side the results for the upper $\Lambda$-doublet state as the initial state are given. The upper part concerns the transitions within the $\Pi_{3 / 2}$ ladder, whereas the spin-orbit changing transitions are considered in the lower part. In addition, symmetry changing $(e \leftrightarrow f)$ and symmetry conserving ( $e \leftrightarrow e, f \leftrightarrow f)$ transitions are distinguished. It should be noted that the lower $\Lambda$-doublet states studied are all of $e$-symmetry and the upper ones of $f$-symmetry. In the figure also theoretical values from Esposti et al. (1995) obtained in a CC calculation using an ab-initio potential are given. The experimental values are scaled to the calculated ones in such a way that the sum of all cross sections given in Fig. 4 is set equal to the 

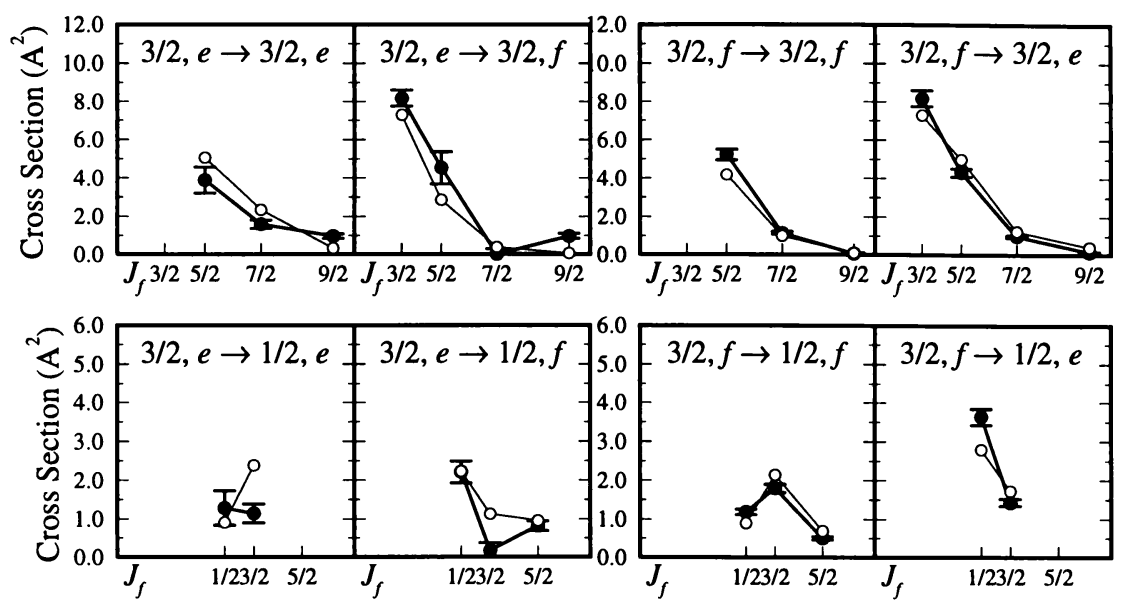

Figure 5. State-to-state cross sections for rotational energy transfer of $\mathrm{OH}$ $\left(\Pi_{3 / 2}, J=3 / 2\right)$ in the upper $(f)$ and lower $(e) \Lambda$-doublet state in collisions with $\mathrm{n}-\mathrm{H}_{2}$. The experimental results $(\bullet)$ are compared with theoretical values (o) from Offer et al. (1994).

sum of the theoretical results. Most striking is the relatively large cross section for the transition $3 / 2, f \rightarrow 5 / 2, e$, in contrast to the much weaker $3 / 2, e \rightarrow 5 / 2, f$ transition. Unexpected was also the relatively weak $\Lambda$ doublet transition. These features are in accordance with the theoretical predictions. Also the other experimental results are in good agreement with theory, apart from the transition $3 / 2, e \rightarrow 5 / 2, e$, which is observed to be much weaker than calculated. The differences between the cross sections for transitions from the upper and the lower doublet state have not been observed before and are caused by interferences of the matrix elements of the intermolecular potential due to the intermediate Hund's case character of $\mathrm{OH}$.

\subsection{2. $\mathrm{OH}-\mathrm{H}_{2}$}

Measurements are performed for both $\mathrm{n}$ (ormal)- $\mathrm{H}_{2}$, with an ortho : para ratio of $3: 1$, and $\mathrm{p}($ ara $)-\mathrm{H}_{2}$. The results for $\mathrm{n}-\mathrm{H}_{2}$ are presented in Fig. 5. Strong differences with He scattering can be seen. First, contrary to He scattering the $\Lambda$-doublet transition is now preferred above all other transitions. Secondly, transitions to the $5 / 2$ state do not show any symmetry dependence; the same holds for transitions to the $7 / 2$ state except for $3 / 2, e \rightarrow 7 / 2, f$ with a nearly zero cross section. The cross sections for scattering by $\mathrm{p}-\mathrm{H}_{2}$ are presented in Fig. 6 . The p- $\mathrm{H}_{2}$ beam consists of $\mathrm{H}_{2}$ molecules in both $J=0$ and $J=2$ in a ratio of $2: 1$. Compared to n$\mathrm{H}_{2}$ the $\Lambda$-doublet cross section is considerably smaller and clear symmetry dependences show up with respect to transitions to the $5 / 2$ state. It turns 

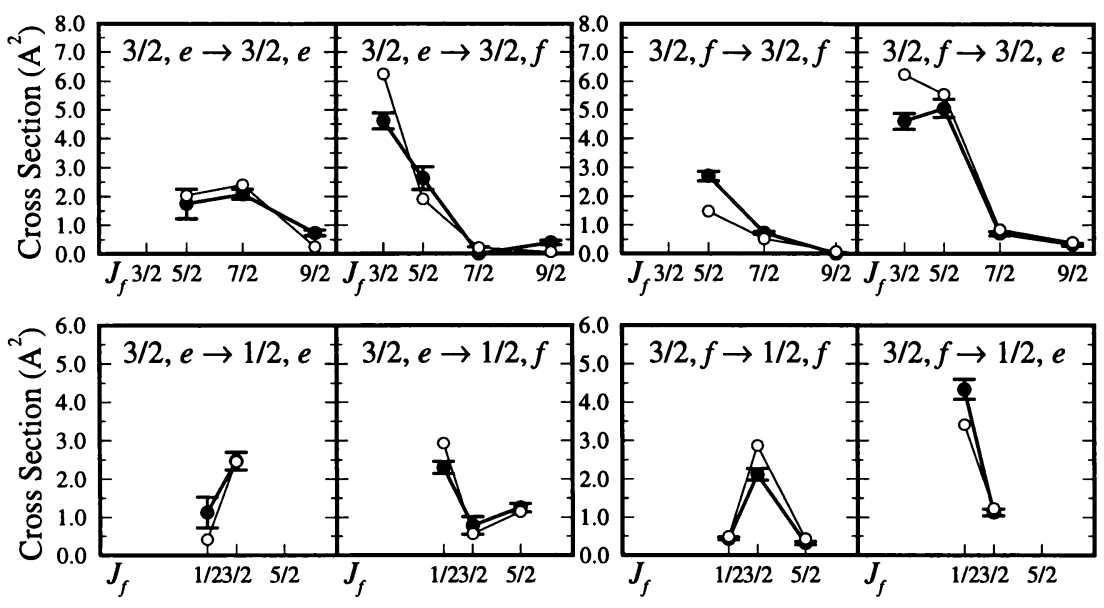

Figure 6. State-to-state cross sections for rotational energy transfer of $\mathrm{OH}$ $\left(\Pi_{3 / 2}, J=3 / 2\right)$ in the upper $(f)$ and lower $(e) \Lambda$-doublet state in collisions with $\mathrm{p}-\mathrm{H}_{2}$. The experimental results $(\bullet)$ are compared with theoretical values (o) from Offer et al. (1994).

out that the $J$ - and parity dependence of the cross sections is in between that for $\mathrm{n}-\mathrm{H}_{2}$ and $\mathrm{He}$. In order to find out what the influence is of the $J=2$ state, measurements were performed with a $\mathrm{p}-\mathrm{H}_{2}$ beam consisting of a $(J=0):(J=2)$ ratio of $1: 1$. In this case the $\Lambda$-doublet cross section increased by a factor of 4 , from which we conclude that the value given in Fig. 6 is determined by collisions with $\mathrm{H}_{2}(J=2)$. In other words a relatively small cross section for the $\Lambda$-doublet transition is expected in case of $\mathrm{H}_{2}(J=0)$ scattering, similar to He. Another strong indication that $\mathrm{H}_{2}(J=0)$ behaves like $\mathrm{He}$ is given by the ratio of the cross sections $\sigma(3 / 2, f \rightarrow 5 / 2, e) / \sigma(3 / 2, f \rightarrow 5 / 2, f)$, which strongly increases when the fraction of $\mathrm{H}_{2}(J=0)$ in the beam increases. In case of He this ratio is 7.3. A comparison to the results of quantum calculations by Offer et al. (1994) based on an ab-initio potential shows a surprisingly good correspondence between theory and experiment. Some deviations are still present, particularly for $\mathrm{p}-\mathrm{H}_{2}$, which probably is due to a lower accuracy of the calculations involving $\mathrm{H}_{2}(J=2)$.

\section{3. $\mathrm{D}_{2} \mathrm{CO}-\mathrm{H}_{2}$}

For $\mathrm{D}_{2} \mathrm{CO}$ differential cross sections were measured at a collision energy of $134 \mathrm{~cm}^{-1}$ for transitions from the upper $1_{1}$ doublet state, $1_{10}$, to the lower doublet state, $1_{11}$, and to the upper and lower $2_{1}$ states, $2_{11}$ and $2_{12}$, respectively. The results are plotted in Fig. 7 as a function of the angle with the beam axis. Also included is the elastic cross section for the 


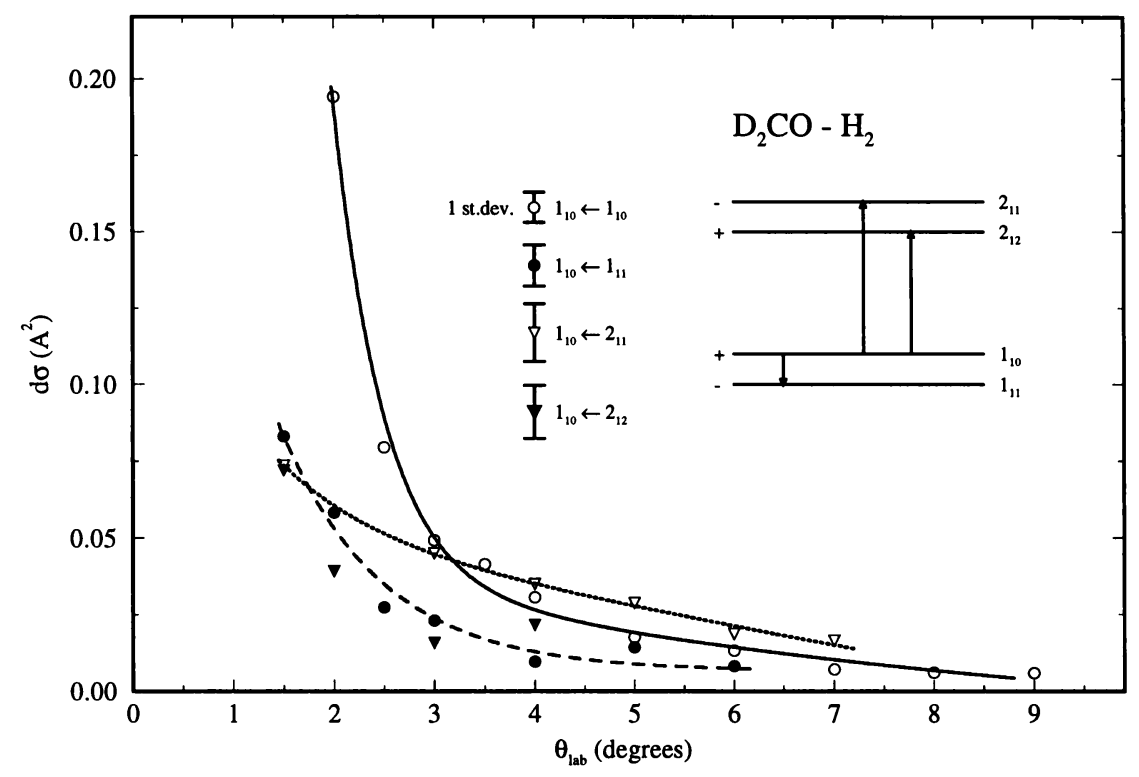

Figure 7. Absolute state-to-state differential cross sections for $\mathrm{D}_{2} \mathrm{CO}\left(1_{10}\right)$ in collisions with $\mathrm{H}_{2}$.

$1_{10}$ state showing a rapid decrease for larger angles. The inelastic cross sections are an order of magnitude smaller at small angles. The total stateto-state cross section is derived by integrating the differential cross section over the full angular range of the scattering. Hereto the sensitivity was, unfortunately, not high enough. However, as can be seen from the figure the differential cross section for the $1_{10} \rightarrow 2_{11}$ transition is systematically larger than the cross sections for the $1_{10} \rightarrow 2_{12}$ and $1_{10} \rightarrow 1_{11}$ transition, which leads to the conclusion that the total cross section $\sigma\left(1_{10} \rightarrow 2_{11}\right)$ is larger than the total cross sections $\sigma\left(1_{10} \rightarrow 2_{12}\right)$ and $\sigma\left(1_{10} \rightarrow 1_{11}\right)$. No calculations on the $\mathrm{D}_{2} \mathrm{CO}-\mathrm{H}_{2}$ system have been reported in literature thus far. Green (1979) has calculated cross sections for $\mathrm{H}_{2} \mathrm{CO}-\mathrm{H}_{2}$ at a collision energy of $200 \mathrm{~cm}^{-1}$, and obtained cross sections which indeed are larger for the rotational transition $1_{1} \rightarrow 2_{1}$ than for the $K$-doublet transition by a factor of 1.6. However, the calculated cross sections $\sigma\left(1_{10} \rightarrow 2_{11}\right)$ and $\sigma\left(1_{10} \rightarrow 2_{12}\right)$ were found to be equal, which deviates from the experiment. Since the rotational spacings of $\mathrm{H}_{2} \mathrm{CO}$ and $\mathrm{D}_{2} \mathrm{CO}$ in the $K_{-1}=1$ ladder differ by not more than $20 \%$, this discrepancy can most likely be ascribed to the fact that only $\mathrm{H}_{2}(J=0)$ is considered in the calculations. 


\section{Conclusions}

Parity resolved state-to-state cross sections are obtained for the rotational energy transfer of $\mathrm{OH}, \mathrm{NH}_{3}$ and $\mathrm{D}_{2} \mathrm{CO}$ in collisions with $\mathrm{He}$ and $\mathrm{H}_{2}$. For $\mathrm{OH}$ and $\mathrm{NH}_{3}$ the results are in good agreement with theory. Some discrepancies are still present, particularly with respect to the predicted parity propensities in $\mathrm{NH}_{3}-\mathrm{He}$, which are not observed in the experiment. The overall good agreement between theory and experiment justifies an extrapolation of the calculations to the lower collision energies in the interstellar space.

\section{Acknowledgements}

The author gratefully acknowledges the $\mathrm{PhD}$ students of the Department of Molecular and Laser Physics who have contributed so much to the success of the collision research, involving particularly Dr. Niek van Hulst, Dr. Jean Schleipen and Dr. Koen Schreel. The skillful technical assistance of Mr. Eugène van Leeuwen is greatly appreciated.

\section{References}

Andresen, P., Aristov, N., Beushausen, V., Husler, D., Lülf, H.W. 1991, J.Chem.Phys. 95,5763

Ebel, G., Krohne, R., Meyer, H., Buck, U., Schinke, R., Seelemann, T., Andresen, P., Schleipen, J., ter Meulen, J.J., Diercksen, G.H.F. 1990, J.Chem.Phys. 93, 6419

Esposti, A.D., Berning, A., Werner, H.J. 1995, J.Chem.Phys. 103, 2067

Green, S. 1979, J.Chem.Phys. 70, 816

Meyer, H., Buck, U., Schinke, R., Diercksen, G.H.F. 1986, J.Chem.Phys. 84, 4976

Offer, A.R., Flower, D.R. 1989, J.Phys.B 22, L439; ibid. 1990, J.Chem.Soc.Far.Trans. 86, 1659

Offer, A.R., van Hemert, M.C., van Dishoeck, E.F. 1994, J.Chem.Phys. 100, 362

Schleipen, J., ter Meulen, J.J. 1991, Chem.Phys. 156, 479

Schleipen, J., ter Meulen, J.J., van der Sanden, G.C.M., Wormer, P.E.S., van der Avoird, A. 1992, Chem.Phys. 163, 161

Schleipen, J., ter Meulen, J.J., Offer, A. 1993, Chem.Phys. 171, 347

Schreel, K., Schleipen, J., Eppink, A., ter Meulen, J.J. 1993, J.Chem.Phys. 99, 8713

Schreel, K., ter Meulen, J.J. 1996, J.Chem.Phys. to be published

Van der Sanden, G.C.M., Wormer, P.E.S., van der Avoird, A., Schleipen, J., ter Meulen, J.J. 1995, J.Chem.Phys. 103, 10001

Van der Sanden, G.C.M., Wormer, P.E.S., van der Avoird, A., Schleipen, J., ter Meulen, J.J. 1992, J.Chem.Phys. 97, 6460

Van Hulst, N.F., ter Meulen J.J., and Dymanus, A. 1987a, J.Chem.Phys. 86, 1407; ibid. 86,4461

Van Hulst, N.F., ter Meulen J.J., and Dymanus, A. 1987b, J.Chem.Phys. 87, 2750 


\section{Discussion}

Herbst: What is the collision energy?

Ter Meulen: The collision energy for both the $\mathrm{NH}_{3}$ and the $\mathrm{OH}$ scattering is about $600 \mathrm{~cm}^{-1}$. It is not easy to do these experiments at collision energies which are similar to those in interstellar sources. In order to obtain cross sections at those low energies one should extrapolate the data by making use of quantum calculations. The good agreement between the present experimental results and theory shows that nowadays these calculations can be performed up to a high degree of reliability.

Kurtadikar: Has the experiment the facility to vary the temperature during collisions? How is it done and what is the range of temperature variation that can be achieved? What are the pressure limitations?

Ter Meulen: It is possible to vary the temperature by choosing different seeding gases, like Xe or $\mathrm{Ne}$, as we did for $\mathrm{H}_{2} \mathrm{CO}-\mathrm{H}_{2}$. In combination with $\mathrm{H}_{2}$ cooling we could vary the temperature between 100 and $900 \mathrm{~K}$. Lower temperatures can be obtained by reducing the angle between the two beams, which, however, requires a complicated reconstruction of the molecular beam machine. The pressure in the collision area is very low $\left(\sim 1.10^{-6} \mathrm{mbar}\right)$ and does not influence the temperature.

Wilson: In astrophysical situations one of every five collision partners are Helium atoms.

Ter Meulen: Since collisions of $\mathrm{NH}_{3}$ with $\mathrm{H}_{2}$ do not create a population inversion between the $3_{3}$ states - at least not at our collision energy - one should consider collisions with He atoms as a possible pumping mechanism. Although there is still a disagreement between experiment and theory about the excitation to the lower $3_{3}$ doublet state, it is clear that there is a strong preference for transitions to the upper $3_{3}$ state. So for He a population inversion can be expected.

Irvine: As was pointed out this morning for cometary studies we need cross sections with $\mathrm{H}_{2} \mathrm{O}$. What are the prospects for obtaining these?

Ter Meulen: $\mathrm{H}_{2} \mathrm{O}-\mathrm{H}_{2}$ might be the next system which we will study. There is a big need for these cross sections, and ab-initio potentials seem to be available now. 